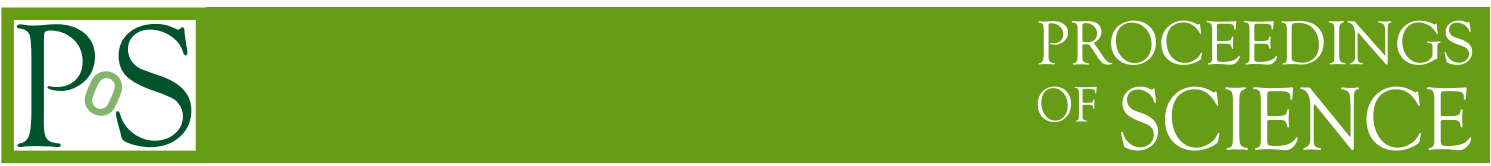

\title{
The extension of the Telescope Array experiment
}

\author{
Eiji Kido $^{*, a}$ for the Telescope Array Collaboration ${ }^{\dagger}$ \\ ${ }^{a}$ Institute for Cosmic Ray Research, University of Tokyo \\ E-mail: ekidodicrr.u-tokyo.ac.jp
}

TA $\times 4$, Telescope Array Low Energy extension (TALE) and the Non-Imaging CHErenkov Array (NICHE) are extension plans of the Telescope Array (TA) experiment. The TA experiment was originally designed to study ultra-high energy cosmic rays with energies above about $1 \mathrm{EeV}$ (1 $\mathrm{EeV}=10^{18} \mathrm{eV}$ ) using surface detectors (SDs) and fluorescence detectors (FDs). These extension plans will enable us to observe cosmic rays with energies over 5 orders-of-magnitude (1 PeV - $100 \mathrm{EeV}$ ) in the same observation site in Utah. The construction of the detectors of $\mathrm{TA} \times 4$, TALE and the prototype of NICHE (j-NICHE) has been already started. New SDs and FDs will be constructed for TA $\times 4$ to cover 4 times larger area than TA to observe cosmic rays with energies above $10 \mathrm{EeV}$ with high statistics. This project is expected to clarify the source of the hotspot in the arrival directions of cosmic rays with energies above $57 \mathrm{EeV}$. The TALE experiment has already measured cosmic rays to well below $0.1 \mathrm{EeV}$ using newly developed FDs. New SDs are being constructed to be added around TALE FDs for better efficiency and resolution. The NICHE detectors are designed to observe cosmic rays with energies above $1 \mathrm{PeV}$ using non-imaging Cherenkov technique. The measurement by TALE and NICHE over wide range of energies will provide accurate understandings of the transition from galactic cosmic rays to extragalactic cosmic rays using cross-calibration between different techniques. The current status and the future prospects of the extension plans are shown here.

38th International Conference on High Energy Physics

3-10 August 2016

Chicago, USA

\footnotetext{
*Speaker.

${ }^{\dagger}$ Full author list and acknowledgements: http://www.telescopearray.org/images/papers/ICRC2015-authorlist.pdf
} 


\section{The TA $\times 4$ experiment}

Telescope Array (TA) is the largest cosmic-ray observatory in the Northern Hemisphere. The TA experiment has 507 surface detectors (SDs) on a square grid with $1.2 \mathrm{~km}$ spacing covering approximately $700 \mathrm{~km}^{2}$. The SDs are surrounded by three fluorescence detector (FD) stations (12, 12 and 14 telescopes). The duty cycle of the SD array is greater than 95\% throughout 5-year observation period, whereas the FD duty cycle is about $10 \%$ because the data is taken only on moonless clear nights. The left figure of Fig.W is the map with locations of the detectors of TA. The latitudes and the longitudes of the locations of the detectors are around $39.30^{\circ} \mathrm{N}$ and $112.91^{\circ} \mathrm{W}$ in Utah in the USA.

The hotspot was observed in the arrival directions of 72 cosmic rays with energies above 57 $\mathrm{EeV}$ [四]. The data used in the analysis was obtained by observing with TA SD for 5 years. The chance probability to exceed the obtained maximum significance $(5.1 \sigma)$ in an isotropy sky is estimated to be $3.4 \sigma$ in the published paper.

$\mathrm{TA} \times 4 \mathrm{SD}$ array was designed to study cosmic rays with energies especially above $57 \mathrm{EeV}$. The spacing of the array is $2.08 \mathrm{~km}$. $500 \mathrm{TA} \times 4$ SDs are planned to cover about 3 times larger area than 507 TA SDs and the combined coverage of TA $\times 4$ SDs with TA SDs is about $3000 \mathrm{~km}^{2}$. This coverage is comparable to that of the Pierre Auger Observatory which has the largest coverage in the Southern Hemisphere.

$25 \%$ energy resolution, 2.2 degree angular resolution and 95\% reconstruction efficiency with $\mathrm{TA} \times 4 \mathrm{SD}$ array for cosmic rays with energies above $57 \mathrm{EeV}$ are expected using simulation studies.

The basic design of TA $\times 4$ SD is same as TA SD []. There are 2 layers of $1.2 \mathrm{~cm}$ thick, $3 \mathrm{~m}^{2}$ plastic scintillators. The scintillation light is read out by wave length shift (WLS) fibers and PMTs. The arrangement of WLS fibers was changed from TA SD. About $67 \%$ of WLS fibers is reduced from TA SD, because the quantum efficiency of PMTs (R8619 Hamamatsu) in TA $\times 4$ SD is about twice as large as that in TA SD. $173 \mathrm{TA} \times 4$ SDs were assembled in Japan and will be finally assembled in the observation site in the near future. $100 \mathrm{TA} \times 4$ SDs were already transported to the observation site.

\section{TALE}

The TALE SD will cover $70 \mathrm{~km}^{2}$ with 103 SDs in the FOV area of the TALE FDs. There are three different detector spacing. The closer look at around the TALE site is the top right figure in Fig.W. We plan to install 40 SDs with $400 \mathrm{~m}$ spacing, and 36 SDs with $600 \mathrm{~m}$ spacing. In the connecting area between the TALE and the TASD array, 27 detectors will be installed with $1.2 \mathrm{~km}$ spacing. The green points in Fig. 1 are the locations of TALE SDs and the red points are the locations of TA SDs. Based on simulation studies, this air shower array has $100 \%$ detection efficiency for energies above $1.4 \times 10^{17} \mathrm{eV}$, and hybrid observations with FDs provides a remarkable improvement in the Xmax determination achieving the error of $20 \mathrm{~g} / \mathrm{cm}^{2}$ comparing with the monocular FD resolution of $40 \mathrm{~g} / \mathrm{cm}^{2}$.

The TALE experiment has already measured cosmic rays to well below $0.1 \mathrm{EeV}$ using newly developed 10 additional telescopes. 35 SDs were already deployed in the site with $400 \mathrm{~m}$ spacing. The same design of SD as TA $\times 4$ SD will be deployed in the rest of sites in 2017 . 

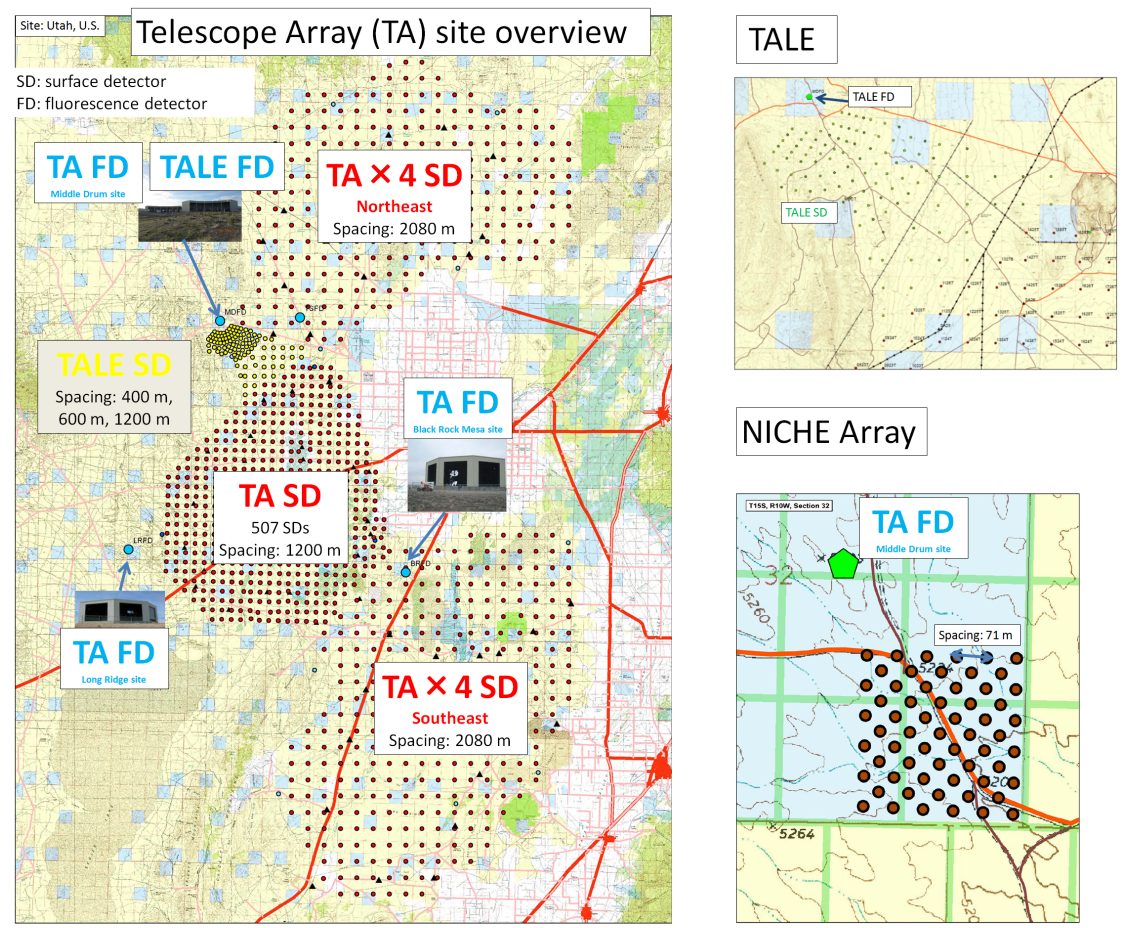

NICHE Array

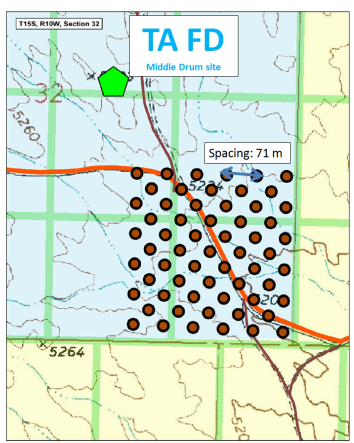

Figure 1: The overview of the TA site is in the left figure. The closer look at around the TALE site is the top right figure. The even closer map around the TA and TALE FD site is the bottom right figure. The locations of NICHE are brown points in this figure.

\section{NICHE}

NICHE was designed to observe cosmic rays with energies down to $10^{15} \mathrm{eV}$ [3]. NICHE detectors observe Cherenkov light and the cosmic rays with energies $1-100 \mathrm{PeV}$ will be reconstructed using both the Cherenkov light Lateral Distribution and the Cherenkov Time Width Lateral Distribution. These two methods will allow energies of cosmic rays and Xmax to be determined. Constructing 15 NICHE detectors are already funded and started as j-NICHE. The location of NICHE is shown in the bottom right figure in Fig.W. The brown points in Fig.W are the locations of the NICHE detectors. The j-NICHE detectors will be deployed at the 15 locations of them.

\section{Future prospect}

The construction of the first $173 \mathrm{TA} \times 4$ SDs is in progress. We try to clarify the source of the hotspot in the arrival directions of cosmic rays with energies especially above $57 \mathrm{EeV}$ with these new SDs. If the full operation of $500 \mathrm{TA} \times 4$ SDs starts, we will obtain about 4 times larger statistics in this energy range than using only TA SDs. 
The TALE experiment has already measured cosmic rays to well below $0.1 \mathrm{EeV}$ using FDs. 35 SDs were already deployed in the site in the FOV of the FDs with $400 \mathrm{~m}$ spacing. The construction of 70 SDs was funded and will be deployed in the TALE site in 2017. Especially the improvement in the composition studies for above $10^{17} \mathrm{eV}$ will be provided by SD and FD hybrid detectors of TALE.

$15 \mathrm{j}$-NICHE detectors were funded and the construction was started. Cosmic rays with energies down to about $10^{15} \mathrm{eV}$ will be observed in the near future.

\section{Acknowledgments}

TA $\times 4$ is supported by the Japan Society for the Promotion of Science through Grants-inAid for Scientific Research on Specially Promoted Research (15H05693). TALE is supported by the Japan Society for the Promotion of Science through Grants-in-Aid for Scientific Research (S) (15H05741). j-NICHE is supported by the Japan Society for the Promotion of Science through Grants-in-Aid for Young Scientists (A) (26707011). The Telescope Array experiment is supported by the aid from the University of Tokyo, the Inter-University Research Program of the Institute for Cosmic Ray Research, and Scientific Research (S) (19104006); by the U.S. National Science Foundation awards PHY-0307098, PHY-0601915, PHY-0649681, PHY-0703893, PHY-0758342, PHY-0848320, PHY-1069280, PHY-1069286, PHY-1404495 and PHY-1404502; by the National Research Foundation of Korea (2007-0093860, R32-10130, 2012R1A1A2008381, 2013004883); by the Russian Academy of Sciences, RFBR grants 11-02-01528a and 13-02-01311a (INR), IISN project No. 4.4502.13, and Belgian Science Policy under IUAP VII/37 (ULB). The foundations of Dr. Ezekiel R. and Edna Wattis Dumke, Willard L. Eccles, and George S. and Dolores Doré Eccles all helped with generous donations. The State of Utah supported the project through its Economic Development Board, and the University of Utah through the Office of the Vice President for Research. The experimental site became available through the cooperation of the Utah School and Institutional Trust Lands Administration (SITLA), U.S. Bureau of Land Management, and the U.S. Air Force. We also wish to thank the people and the officials of Millard County, Utah for their steadfast and warm support. We gratefully acknowledge the contributions from the technical staffs of our home institutions. An allocation of computer time from the Center for High Performance Computing at the University of Utah is gratefully acknowledged.

\section{References}

[1] R. Abbasi et al. ApJ. 790, L21 (2014).

[2] T. Abu-Zayyad et al. NIM-A, 689, 87 (2012).

[3] D.R. Bergman, J.F. Krizmatnic, Y. Tsunesada, Proceedings of the 34th ICRC (Hague) PoS(ICRC2015)635. 